\section{Variations in the Insecticide Tolerance of Insects}

RECENT communications ${ }^{1,2}$ on the variance of the dosage-mortality response of strains of housefly and mosquito susceptible and resistant to insecticide serve to emphasize that, although there is considerable information on the inheritance of resistance to insecticide in some insects, especially houseflies ${ }^{3}$, Drosophila ${ }^{4}$ and now mosquitoes ${ }^{5}$, there has as yet been no direct estimate of how much of the unselected tolerance is genetically determined, how much is due to environmental effects. Beard, ${ }^{6}$, who first directed attention to the possibilities, considered the non-genetic variation might so predominate as to overwhelm the genetic components.

One would not wish to dispute the contention ${ }^{1}$ that insect populations comprised of a single resistancesusceptibility genotype are likely to be less variable in their tolerances than are populations containing all possible resistance-susceptibility genotypes. But that fact is scarcely relevant to what Hewlett originally suggested? namely, that a portion of the tolerance variance is susceptible to manipulation without any change in gene frequency. That this interpretation was, at least in part, intended is shown by the example selected ${ }^{8}$, the decrease in variance of the tolerance found with a DDT-resistant strain of housefly when increasing amounts of the insecticidally inert synergist DMC were added to the applied DDT. One point which strongly supports Hewlett's hypothesis, but has not been commented upon, is the very obvious increase in variance with the smallest amount of DMC compared with that found with DDT alone ${ }^{8}$.

It is usual to assume that the genes for resistance are extremely rare in Nature, or, as stated by Wilson. Jones and Davidson ${ }^{1}$, so infrequent that an average sample of wild insects is unlikely to contain resistant genes. This assumption has been made repeatedly in attempts to explain the often rather slow emergence of resistance in the field, but, as was recently pointed out $^{9}$, it is unlikely that this assumption of rarity is correct. Indeed, the only quantitative work as yet available on the frequency of resistant genes in wild populations showed that there may be as many as 12 per cent of dieldrin-resistant heterozygotes in the Anopheles gambiae populations of areas as yet untouched by dieldrin ${ }^{10}$. Finally, laboratory populations originate as samples from wild populations, most but not all attempts in the laboratory to induce insecticide resistance are successful, and sometimes resistance to a number of insecticides can be developed sequentially ${ }^{11}$.

\section{SPILLER}

Plant Diseases Division,

Department of Scientific and Industrial Research, Auckland, Now Zealand.

${ }^{1}$ Wilson-Jones, K., and Davidson, G., Nature, 182, 403 (1958).

${ }^{2}$ Hewlett, P. S., Nature, 182, 404 (1958).

${ }^{3}$ Milani, R., Riv. Parassit., 17, 233 (1956): 18, 43 (1957).

- Crow, J. F. "Annual Review of Entomology”, 2, 227 (1957).

s Davidson, G., Bull. Wld. Hlth. Org., 18, 579 (1958).

${ }^{6}$ Beard, R. L., Science, 115, 608 (1952).

' Hewlett, P. S., Ann. App. Biol., 46, 37 (1958).

${ }^{8}$ Perry, A. S. Mattson, A. M., and Buckner, A. J., Biol. Bull., Wood, Hole, 104, 426 (1953)

${ }^{9}$ Spiller, D., Presidential Address, N.Z. Entomological Society, 1958 (in the press).

10 Brown, A. W. A., Bull. Wld. Blth. Org., 18, 309 (1958).

11 Metcalf, R. L., "Organic Insecticides" (Interscience Publishers, Inc New York, 1955).

\section{Fluorescent Mast-Cell Reaction in Precancerous Skin of the Lizard Lacerta agilis}

THE mast-cell reaction of the mouse skin, painted with tar or carcinogenic hydrocarbons, has been known for a long time ${ }^{1}$. According to several authors, these cells show a golden-brown fluorescence when examined under the microscope in ultra-violet light ${ }^{2}$, though other investigations give some evidence to the contrary ${ }^{3}$.

Recently, it has been established that the mast cells of the mouse and the rat contain not only hist.amine but also 5-hydroxytryptamine*. This sub. stance can be converted into a fluorescent $\beta$-carboline derivative by the action of formaldehyde, and in the mast cells of the precancerous mouse skin it reaches a concentration which is normally only achieved by the cells of the enterochromaffin system ${ }^{5}$.

In the mastocytosis of the mouse skin after painting with 9,10-dimethyl-1,2-benzanthracene in acetone it could be demonstrated to direct assay and by paper chromatographic examination that the fluorescent substance of the mastocytes is the 5-hydroxytryptamine derivative $\beta$-carboline ${ }^{2}$.

Continuing these investigations, we examined a cutaneous carcinoma of a lower vertebrate, namely, the squamous-cell carcinoma of the lizard Lacerta agilis. This tumour seemed pre-eminently suitable for this purpose, because a distinct precancerous phase occurs in its development ${ }^{6,7}$. At first we had seven tumour-bearing animals, but by extending the material we finally had 104 specimens at our disposal.

From these specimens the precancerous portions of the skin were excised. The examination of the mast cells was carried out with frozen sections (cut in a horizontal as well as in a vertical direction), freehand sections and tissue spreads prepared from dermal scrapings. The skin material, fixed in 10 per cent formalin or otherwise, was examined under the microscope in ultra-violet light. Then the material was stained either with acetylated sudan black for phospholipids $^{8}$ or with toluidine blue for mast cells.

Just as in the mouse, the mast cells of the precancerous skin of the lizard gave a golden-brown fluorescence in ultra-violet light, but only after treatment with formalin. The fact that a nonfluorescent precursor substance can be extracted from the mast cells by prolonged treatment of the skin tissue with acetone suggests that the fluorescence is a consequence of the action of formaldehyde on the above-mentioned substance. Acetone treatment apparently did not affect the ability of the mast cell granules to stain with either acetylated sudan black or with toluidine blue.

To establish the chemical identity of the fluorescing substance we prepared an extract of the precancerous lizard skin. For this purpose the dermal scrapings of the skins were homogenized in acetone, after which the extract was concentrated in vacuo. After the fats had been removed with petroleum ether the extract was treated with formaldehyde and could then be examined in ultra-violet light. The extract treated with formaldehyde exhibits the same goldenbrown fluorescence as was found with the extract of mouse skin prepared in the same way ${ }^{2}$. A further extraction of the skin fragments with fat solvents failed to yield additional fluorescent material. 